\title{
Associations between plasma concentrations of lenvatinib and angiopoietin and clinical responses to lenvatinib therapy in Japanese patients with thyroid cancer
}

\section{Maho Kumagai}

Akita University Hospital

Mitsuji Nagahama

Ito Hospital

Yumiko Akamine

Akita University Hospital

Tomoko Ozeki

Akita University Hospital

Akifumi Suzuki

Ito Hospital

Kiminori Sugino

Ito Hospital

Koichi Ito

Ito Hospital

Masatomo Miura ( $\nabla$ m-miura@hos.akita-u.ac.jp )

Akita University Hospital

\section{Research Article}

Keywords: lenvatinib, angiopoietin-1, angiopoietin-2, plasma concentration, response

Posted Date: October 14th, 2021

DOl: https://doi.org/10.21203/rs.3.rs-828572/v2

License: (c) (i) This work is licensed under a Creative Commons Attribution 4.0 International License. Read Full License 


\section{Abstract}

The purpose of this study was to investigate the relationships among plasma concentrations $\left(\mathrm{C}_{0}\right)$ of lenvatinib, angiopoietin (Ang)-1 and Ang-2, and clinical responses to lenvatinib therapy in thyroid cancer patients. The median change rates of Ang-1 and Ang-2 at 1 month after treatment from baseline in all patients were $-15.3 \%$ and $-48.4 \%$, respectively. However, the change of Ang- 1 and Ang-2 at 1 month from baseline did not correlate with lenvatinib $C_{0}$. In patients with partial response $(P R)$ and stable disease to lenvatinib, Ang-2 at 1 month were significantly lower than Ang-2 at baseline $(P<0.001$ and $P<$ 0.05 , respectively), but were not significantly lower in patients with progressive disease. The area under the ROC for PR prediction was 0.667 , giving the best sensitivity $(69.2 \%)$ and specificity $(73.9 \%)$ at a threshold of the change rate of Ang- 2 of $-49.83 \%$. In patients who continued treatment with lenvatinib for 1 year, Ang-2 at 1 month and 1 year were significantly lower than those at baseline (each $P<0.001$ ). The change of Ang-2 at 1 month after treatment from baseline rather than simply the Ang-2 level at baseline may be important as a biomarker of the inhibitory effect of angiogenesis by lenvatinib.

\section{Introduction}

Lenvatinib is an oral inhibitor of multiple tyrosine kinase receptors, including vascular endothelial growth factor (VEGF) receptors $1-3$, fibroblast growth factor receptors $1-4$, platelet-derived growth factor receptor alpha, stem cell factor receptor and rearranged during transfection ${ }^{1-4}$, and it is approved for the treatment of thyroid cancer. VEGF receptors 1-3 are expressed on endothelial cells and play important roles in both physiologic and pathologic angiogenesis ${ }^{5}$, and the angiogenesis induced by overexpressed VEGF has been reported to be significantly suppressed by treatment with lenvatinib ${ }^{4}$.

Angiopoietin-1 (Ang-1) and angiopoietin-2 (Ang-2) are ligands for the endothelial-specific receptor tyrosine kinase $\mathrm{Tie} 2^{6}$. VEGF and Ang-2 are expressed early in tumor formation, and their levels increase throughout tumor growth ${ }^{7}$. Ang-2 facilitates VEGF-induced angiogenesis and is expressed during vascular remodeling ${ }^{8}$. On the other hand, changes in the expression of Tie2 and Ang-1 are not observed throughout tumor growth ${ }^{7}$. Previously, baseline levels of Ang-2 prior to lenvatinib therapy in patients with advanced medullary or differentiated thyroid cancer have been reported to be predictive of clinical outcomes of lenvatinib ${ }^{9,10}$. Namely, low baseline levels of Ang-2 before lenvatinib therapy are associated with tumor reduction and prolonged progression-free survival (PFS) 9,10. Therefore, Ang-2 may be predictive of sensitivity to lenvatinib.

Relationships between plasma concentrations and efficacy or toxicity of oral targeted antineoplastic drugs have been studied intensely ${ }^{11,12}$. The target plasma trough concentration $\left(C_{0}\right)$ of lenvatinib for patients with thyroid cancer is approximately $51.5 \mathrm{ng} / \mathrm{mL}$ based on the mean $\mathrm{C}_{0}$ at the steady-state in a phase 3 trial ${ }^{11,13}$. In our previous study, we demonstrated that the target lenvatinib $C_{0}$, as the threshold between the $C_{0}$ and optimal response, lies within a range from 42 to $88 \mathrm{ng} / \mathrm{mL}^{14}$. Therefore, Ang-2 levels are reduced as part of the inhibitory effect of angiogenesis by lenvatinib, and plasma concentration of 
lenvatinib may relate to the decreasing of Ang-2 from baseline. However, the associations between plasma concentration of lenvatinib and Ang-2 have not yet been clarified.

In the present study, then, we aimed to retrospectively examine the relationships between plasma concentrations of lenvatinib and Ang-2, and the impacts of these concentrations on clinical responses to lenvatinib therapy in Japanese patients with thyroid cancer.

\section{Results}

Median Ang-1 and Ang-2 levels at 1 month after initiation of lenvatinib therapy for 36 patients were significantly lower than those pre-therapy (Ang-1: $5750 \mathrm{pg} / \mathrm{mL}$ from $6459 \mathrm{pg} / \mathrm{mL}, P<0.001$; Ang-2: 967 $\mathrm{pg} / \mathrm{mL}$ from $1590 \mathrm{pg} / \mathrm{mL}, P<0.001)$. The median rates of change of Ang-1 and Ang-2 levels at 1 month from baseline in 36 patients were $-15.3 \%$ and $-48.4 \%$, respectively. However, the rates of change of Ang1 and Ang-2 levels at 1 month relative to baseline did not correlate with the $C_{0}$ for lenvatinib at 1 month after initiation of treatment (Fig. 1). On the other hand, there were no significant differences in Ang-1 or Ang-2 levels between 1 month and 1 year time points ( $P=0.267$ and 0.248 , respectively).

Partial response (PR), stable disease (SD) and progressive disease (PD) were observed as best responses to lenvatinib treatment in 23, 8 and 5 patients, respectively (Table 1). Among groups with these best responses, there were no significant differences in Ang-1 plasma levels at baseline or at 1 month and 1 year after initiation of lenvatinib therapy, and there were no significant differences in the rate of change of Ang-1 at 1 month and 1 year from baseline (Table 1). However, in patients with PR to lenvatinib, Ang-1 levels 1 month after treatment initiation were significantly lower than Ang-1 levels at baseline $(P<0.01$, Table 1).

Similar to the Ang-1 levels, there were no significant differences in Ang-2 levels at baseline or at 1 month and 1 year after initiation of lenvatinib therapy among patients with PR, SD and PD; however, in patients with PR to lenvatinib, Ang-2 levels at 1 month and 1 year after treatment initiation were significantly lower than Ang-2 levels at baseline $(P<0.001$ and $P<0.01$, respectively, Table 1$)$.

There were no significant differences in lenvatinib $\mathrm{C}_{0} 1$ month after treatment initiation among patients with $\mathrm{PR}, \mathrm{SD}$ and $\mathrm{PD}$ (Table 1); however, in patients with $\mathrm{PR}$, lenvatinib $\mathrm{C}_{0}$ at 1 year after treatment initiation was significantly lower than at 1 month $(P<0.01)$.

A waterfall plot of rate of change of Ang-2 levels 1 month after initiation of lenvatinib therapy relative to baseline is shown in Fig. 2. The rate of change of Ang-2 levels in 2 patients ( 1 patient with papillary thyroid cancer and 1 patient with follicular thyroid cancer) increased after lenvatinib therapy, and these 2 patients showed an overall response of PD for lenvatinib therapy.

A receiver operating characteristic (ROC) analysis showed the discrimination potential of the rate of change of Ang-2 levels for prediction of PR to lenvatinib (Fig. 3). The area under the ROC was 0.667 (95\% 
confidence interval $(\mathrm{Cl}), 0.478-0.873)$, giving the best sensitivity $(69.2 \%)$ and specificity $(73.9 \%)$ at a threshold of

Patients were divided into two groups depending on their exhibited rates of change of Ang-2 levels: those with rates of change of at least $-49.83 \%$ and those with rates of change of less than $-49.83 \%$. The overall survival (OS) rates of patients in the group with changes of at least $-49.83 \%$ tend to be longer than those with changes less than $-49.83 \%$, but these differences were not statistically significant (median OS: 676 day and 273 day, respectively, Fig. 4). The median 1 year OS for patients having rates of change of levels of Ang- 2 of at least $-49.83 \%$ and less than $-49.83 \%$ were $62.5 \%$ and $45 \%$, respectively.

Furthermore, the 18 patients that continued treatment with lenvatinib for 1 year were compared with 18 patients who discontinued treatment at less than 1 year (Table 2). There were no significant differences between Ang-1 and Ang-2 levels at baseline or at 1 month after initiation of lenvatinib therapy. The rates of change of Ang-1 and Ang-2 levels at 1 month relative to baseline between these 2 groups were also not significantly different. In addition, there was no significant difference in lenvatinib $\mathrm{C}_{0}$ at 1 month after initiation of treatment between these 2 groups (Table 2).

However, in patients who continued lenvatinib treatment beyond 1 year, Ang-2 levels and the Ang-2/Ang-1 ratio at 1 month and 1 year after treatment initiation were significantly lower than those at baseline (each $P<0.001$, Table 2). In addition, lenvatinib $C_{0}$ at 1 year after treatment initiation was significantly lower than that at 1 month $(P<0.01)$. On the other hand, in patients who discontinued lenvatinib therapy prior to 1 year, both Ang-1 and Ang-2 levels 1 month after treatment were significantly lower than those at baseline $(P<0.01$ and $P<0.05$, respectively).

None of the 10 patients with anaplastic thyroid cancer continued treatment with lenvatinib for more than 1 year ( $P=0.002$ in Table 2$)$. Therefore, subgroup analyses were performed only on the 21 patients with papillary thyroid cancer (PTC) or follicular thyroid cancer (FTC) (Table 2, lower berth). Similar to the results from the 36 patients of five different histological types of thyroid cancer, there were no significant differences in Ang-1 and Ang-2 levels at baseline or at 1 month after initiation of lenvatinib therapy. Similarly, there were no significant differences in the rates of change at 1 month relative to baseline between these 2 groups. However, Ang-2 levels at 1 month after treatment initiation were significantly lower than Ang-2 at baseline $(P<0.001)$.

\section{Discussion}

In the present study, we found that Ang-2 levels were significantly reduced by the administration of lenvatinib. Especially in patients that achieved PR to lenvatinib therapy, Ang-2 levels were significantly decreased. A cut-off value for the rate of change of Ang-2 from baseline to attain PR for lenvatinib therapy was $-49.83 \%$ according to the ROC analysis. By monitoring Ang-2 levels at 2 sampling points, before initiation of therapy and at 1 month after initiation, we might be able to predict clinical outcomes for lenvatinib. However, a prediction of efficacy for lenvatinib using Ang-2 levels may only be possible 
until 1 year after initiation of lenvatinib administration. In the present study, we found that Ang-2 levels at 1 year tend to increase compared with those at 1 month (rate of change for patients with PR: $-40.2 \%$ from $-51.8 \%$, that for patients with SD: $-36.9 \%$ from $-39.8 \%$, and for patients continued therapy for 1 year: $-40.2 \%$ from $-51.0 \%$ ). Increased Ang-2 levels after lenvatinib therapy may correlate with tumor progression and OS.

Although Ang-2 levels were significantly reduced by the administration of lenvatinib, Ang-1 levels were not significantly changed by lenvatinib. Therefore, changes of the Ang-2/Ang-1 ratio by lenvatinib depended on the change of Ang-2 levels. Our present study indicated that Ang-2 better predicted the efficacy of lenvatinib in patients with thyroid cancer than did either Ang-1 or the Ang-2/Ang-1 ratio. Previously, Ang-2 levels were reported to be associated with tumor angiogenesis in hepatocellular carcinoma ${ }^{15}$, lung tumors ${ }^{16}$ and colorectal cancers ${ }^{17}$, and the relationships between Ang-2 levels at pre-therapy and clinical outcomes after drug therapy have been investigated ${ }^{10,18,19}$. In a phase 3 study of lenvatinib for thyroid cancers, a significant association between Ang-2 levels at pre-therapy of lenvatinib and clinical outcomes were also observed ${ }^{9,10}$. However, in the present study, there were no significant differences in Ang-2 levels at baseline among patients with PR, SD and PD or among patients who continued lenvatinib therapy longer than 1 year.

Patients with higher Ang-2 levels of 8011 and $7174 \mathrm{pg} / \mathrm{mL}$ at baseline were decreased to 3568 and 3686 $\mathrm{pg} / \mathrm{mL}$, respectively, at 1 month and then 2593 and $3011 \mathrm{pg} / \mathrm{mL}$, respectively, at 1 year after the start of lenvatinib therapy. Thus, the efficacy of PR for lenvatinib in patients with a higher Ang-2 levels at baseline were confirmed. On the other hand, patients with Ang-2 levels of 2280 and $3493 \mathrm{pg} / \mathrm{mL}$ at baseline were increased to 11305 and $11511 \mathrm{pg} / \mathrm{mL}$, respectively, at 1 month after initiation of lenvatinib therapy, and these patients were non-responders to lenvatinib and moved toward PD. Therefore, the rate of change of Ang-2 at 1 month after treatment initiation from baseline rather than a single data point consisting of the Ang-2 level at baseline may be important as a biomarker of the inhibitory effect of angiogenesis by lenvatinib.

In the present study, we demonstrated a lack of significant correlation between the plasma concentrations of lenvatinib and Ang-2. High plasma exposures of lenvatinib therefore seem to be unnecessary to attain clinical responses. The median $C_{0}$ of lenvatinib at 1 year in patients with PR (49.7 $\mathrm{ng} / \mathrm{mL}$ ) was significantly lower than those at 1 month after the initiation of treatment $(71.2 \mathrm{ng} / \mathrm{mL})$. This decrease in $\mathrm{C}_{0}$ over time was caused by the dose reduction of lenvatinib due to the onset of side effects. In addition, the median $\mathrm{C}_{0}$ of lenvatinib at 1 year in patients who continued treatment more than 1 year $(59.7 \mathrm{ng} / \mathrm{mL})$ was significantly lower than those on 1 month after treatment $(82.0 \mathrm{ng} / \mathrm{mL})$. The target $\mathrm{C}_{0}$ of lenvatinib, is optimal point of balance between benefit and toxicity; for patients with thyroid cancer the target $C_{0}$ is reported to be $51.5 \mathrm{ng} / \mathrm{mL}^{11,13}$. The $C_{0}$ of lenvatinib of $51.5 \mathrm{ng} / \mathrm{mL}$ in these previous studies is similar to the median $\mathrm{C}_{0}$ at 1 year after treatment in the present study. 
As shown in Fig. 1, the rate of change of Ang-2 at 1 month after treatment initiation from baseline was decreased to about $44.3 \%$ with a $C_{0}$ of lenvatinib of $51.5 \mathrm{ng} / \mathrm{mL}(y=-0.0352 x-42.533)$. This rate of change of Ang-2 from baseline in the context of a $C_{0}$ of lenvatinib of approximately $51.5 \mathrm{ng} / \mathrm{mL}$ was similar those in higher contexts of $C_{0}$ of lenvatinib of more than $100 \mathrm{ng} / \mathrm{mL}$. Therefore, after beginning treatment with an initial dose of lenvatinib of $24 \mathrm{mg}$, subsequent required doses may be calculated according to the target $C_{0}$ of $51.5 \mathrm{ng} / \mathrm{mL}$ early to avoid adverse events of lenvatinib.

Our current findings may be interpreted within the context of a limitation regarding types of thyroid cancer. Different types of thyroid cancer are described according to histological feature. These types include well-differentiated papillary thyroid cancer, which makes up 75 to $80 \%$ of thyroid cancer cases, follicular thyroid cancer (8 to $10 \%$ ), poorly differentiated thyroid cancer (5 to $7 \%$ ), medullary thyroid cancer (5 to $7 \%$ ) and anaplastic thyroid cancer (2 to $3 \%)^{20}$. Lenvatinib is approved for anaplastic thyroid cancer in Japan and lenvatinib is used for all histological types of thyroid cancer. Therefore, we analyzed data without separating different histological types; however, additional studies in different histological types of thyroid cancer may be necessary.

\section{Conclusion}

The rate of change of Ang-2 levels at 1 month after treatment from baseline did not correlate with the lenvatinib $\mathrm{C}_{0}$ at the same timing; however, Ang-2 levels were significantly reduced by the administration of lenvatinib. In particular, Ang-2 levels in patients with PR to lenvatinib therapy or patients who continued treatment with lenvatinib for 1 year were significantly decreased. The rate of change of Ang-2 at 1 month after treatment from baseline may be important as a biomarker of the inhibitory effect of angiogenesis by lenvatinib.

\section{Materials And Methods}

\section{Patients and protocols}

Thirty-seven Japanese patients receiving treatment with lenvatinib (LENVIMA; Eisai Co., Ltd., Tokyo, Japan) for thyroid cancer at Ito Hospital from January 2016 through December 2018 were consecutively enrolled in this study. One female patient was excluded because of adverse events that occurred shortly after beginning treatment with $24 \mathrm{mg} /$ day lenvatinib. Accordingly, thirty-six patients ( 23 women and 13 men) were analyzed in this study. Thirty-four patients in this study had participated in our previous studies $^{14}$. The mean age was $65 \pm 11$ years, and the mean body weight was $59 \pm 14 \mathrm{~kg}$. There were no patients with serious renal or hepatic dysfunction. The study was approved by the Ethics Committees of Ito Hospital (approval numbers 137 and 330) and Akita University School of Medicine (approval number 790) and was conducted in accordance with the ethical standards of the 1964 Declaration of Helsinki and its later amendments or comparable ethical standards. Patients provided written informed consent for participation in the study. 
The inclusion criteria were in accordance with standard eligibility criteria for lenvatinib treatment ${ }^{21}$. All patients received oral lenvatinib $24 \mathrm{mg}$ once daily as an initial dose. Sequential dose reductions to 20,14 , 10,8 and $4 \mathrm{mg} /$ day were conducted based on the grade of each side effect according to a guide in the package insert ${ }^{21}$. The evaluation of clinical response was determined by computed tomography according to Response Evaluation Criteria in Solid Tumors (RECIST) 1.1 criteria at 1 month after the beginning of lenvatinib treatment, then every 3 months during the first year and every 3 to 6 months during the following 3 years, depending on response to treatment and the clinical condition of the patient. The OS was defined as the time from first lenvatinib administration until death from any cause or until the final follow-up.

At 1 month (range from day 22 to day 36 after administration) and 1 year after lenvatinib therapy, whole blood samples $(5 \mathrm{~mL})$ were collected by venepuncture just before administration $\left(\mathrm{C}_{0}\right)$ of lenvatinib. Plasma was isolated by centrifugation at $1900 \mathrm{~g}$ for $15 \mathrm{~min}$ and was stored at $-40^{\circ} \mathrm{C}$ until analysis. Quantification of lenvatinib $\mathrm{C}_{0}$, Ang-1 and Ang-2 in plasma were performed at the same time. Ang- 1 and Ang-2 values at baseline were analyzed via blood sampling within 1 week before the initiation of lenvatinib therapy.

\section{Analytical Methods}

Lenvatinib $\mathrm{C}_{0}$ was measured by high-performance liquid chromatography (HPLC) and ultraviolet spectroscopic analysis, as previously described ${ }^{14,22}$. The calibration curve generated for lenvatinib in human plasma was linear over the concentration range of 5 to $1000 \mathrm{ng} / \mathrm{mL}$. The limit of quantification of lenvatinib for this assay was $5 \mathrm{ng} / \mathrm{mL}$. The coefficients of variation and accuracies for intra- and interday assays at the concentration range of 5 to $1000 \mathrm{ng} / \mathrm{mL}$ were less than $12.6 \%$ and within $10.6 \%$, respectively. Plasma concentrations of Ang-1 and Ang-2 were assayed using enzyme-linked immunosorbent assays (ELISA) (R\&D Systems Inc., Minneapolis, MN, USA.), following the manufacturer's instructions.

\section{Statistical analyses}

Shapiro-Wilk tests were used to assess distributions. Spearman's rank correlation coefficient values were used to assess correlations between continuous values. Kruskal-Wallis tests were used to compare continuous values for more than three groups, and median values in the different groups were compared using Mann-Whitney $U$ tests with Bonferroni's correction. The Wilcoxon signed-rank test was used to determine differences in continuous values for each patient. Results with $P$ values of less than 0.05 were considered statistically significant in correlation coefficient tests and multiple comparison tests. In contrast, results with $P$ values of less than $0.05 / 3$ were considered statistically significant in comparisons between two groups after comparisons among three groups. An ROC curve was used to determine the best cut-off value for predictive factors, which had a minimum distance from the upper left corner to the 
point on the ROC curve. Survival curve for OS was analyzed using the Kaplan-Meier method. Statistical analyses were performed with SPSS 20.0 for Windows (SPSS IBM Japan Inc., Tokyo, Japan).

\section{Abbreviations}

Ang-1: angiopoietin-1

Ang-2: angiopoietin-2

ATC: anaplastic thyroid cancer

$\mathrm{C}_{0}$ : plasma trough concentration

Cl: confidence interval

ELISA: enzyme-linked immunosorbent assays

FTC: follicular thyroid cancer

HPLC: high-performance liquid chromatography

MTC: medullary thyroid cancer

OS: overall survival

PD: progressive disease

PDTC: poorly differentiated thyroid cancer

PFS: prolonged progression-free survival

PR: partial response

PTC: papillary thyroid cancer

RECIST: Response Evaluation Criteria in Solid Tumors

ROC: receiver operating characteristic

SD: stable disease

VEGF: vascular endothelial growth factor

\section{Declarations}

Acknowledgments 
This work was supported by a grant (number 20K07150) from the Japan Society for the Promotion of Science, Tokyo, Japan.

\section{Funding}

This work was supported by a grant (number 20K07150) from the Japan Society for the Promotion of Science, Tokyo, Japan.

\section{Conflicts of interest/Competing interests}

All authors have no conflicts of interest and have no relevant relationships to disclose.

\section{Ethics approval}

The study was approved by the Ethics Committees of Ito Hospital (approval numbers 137 and 330) and Akita University School of Medicine (approval number 790).

\section{Consent to participate}

Informed consent was obtained from all patients.

\section{Research involving human participants}

This study was performed in accordance with the ethical standards of the Declaration of Helsinki and its subsequent amendments.

\section{Data availability}

Data and material are available on request from the correspondence author.

\section{Code availability}

Not applicable

\section{Authors' contributions}

$M K, M N, Y A, T O, A S, K S, K I$, and MM participated in the design of the study and reviewed the results. MN, AS, KS, and KI were responsible for the collection of patients and were involved in acquisition of data. $M K, Y A$ and MM analyzed plasma concentrations. MK and MM were responsible for the statistical analyses. MK, MN, and MM drafted the manuscript. AS, KS, TO, YA, and KI helped to draft the manuscript. All authors read and approved the final manuscript.

\section{Consent to publish}

Patients signed informed consent regarding publishing their data. 


\section{References}

1. Matsui, J. et al. E7080, a novel inhibitor that targets multiple kinases, has potent antitumor activities against stem cell factor producing human small cell lung cancer $\mathrm{H} 146$, based on angiogenesis inhibition. Int. J. Cancer, 122, 664-671 (2008).

2. Okamoto, K. et al. Antitumor activities of the targeted multi-tyrosine kinase inhibitor lenvatinib (E7080) against RET gene fusion-driven tumor models. Cancer Lett, 340, 97-103 (2013).

3. Tohyama, O. et al. Antitumor activity of lenvatinib (e7080): an angiogenesis inhibitor that targets multiple receptor tyrosine kinases in preclinical human thyroid cancer models. J Thyroid Res 2014, 638747 (2014)

4. Yamamoto, Y. et al. Lenvatinib, an angiogenesis inhibitor targeting VEGFR/FGFR, shows broad antitumor activity in human tumor xenograft models associated with microvessel density and pericyte coverage. Vasc Cell, 6, 18 (2014).

5. Ellis, L. M. \& Hicklin, D. VEGF-targeted therapy: mechanisms of anti-tumour activity. Nat. Rev. Cancer $J, 8,579-591$ (2008).

6. Yancopoulos, G. D. et al. Vascular-specific growth factors and blood vessel formation. Nature, 407 (6801), 242-248 (2000).

7. Tse, V. et al. G. 4th Harsh, The temporal-spatial expression of VEGF, angiopoietins-1 and 2, and Tie-2 during tumor angiogenesis and their functional correlation with tumor neovascular architecture. Neurol. Res. 25, 729-738(2003).

8. Karpanen, T. \& Makinen, T. Regulation of lymphangiogenesis-from cell fate determination to vessel remodeling. Exp. Cell Res, 312 (5), 575-583 (2006).

9. Schlumberger, M. et al. A Phase II Trial of the Multitargeted Tyrosine Kinase Inhibitor Lenvatinib (E7080) in Advanced Medullary Thyroid Cancer. Clin. Cancer Res, 22, 44-53 (2016).

10. Tahara, M. et al. Exploratory analysis of biomarkers associated with clinical outcomes from the study of lenvatinib in differentiated cancer of the thyroid. Eur. J. Cancer, 75, 213-221 (2017).

11. Verheijen, R. B. et al. Practical recommendations for therapeutic drug monitoring of kinase inhibitors in oncology. Clin. Pharmacol. Ther, 102, 765-776 (2017).

12. Mueller-Schoell, A. et al. Therapeutic drug monitoring of oral targeted antineoplastic drugs. Eur. J. Clin. Pharmacol, 77, 441-464 (2021).

13. Food and Drug Administration. Center for Drug Evaluation and Research Lenvatinib Clinical Pharmacology and Biopharmaceutics Review. 2015/2069470rig1s000ClinPharmR.pdf> (2014)

14. Nagahama, M. et al. Association of lenvatinib trough plasma concentrations with lenvatinib-induced toxicities in Japanese patients with thyroid cancer. Med. Oncol, 36, 39 (2019).

15. Scholz, A. et al. Angiopoietin-2 serum levels are elevated in patients with liver cirrhosis and hepatocellular carcinoma. Am. J. Gastroenterol, 102, 2471-2481 (2007).

16. Park, J. H. et al. Serum angiopoietin-2 as a clinical marker for lung cancer. Chest, 132, 200-206 (2007). 
17. Gu, J. et al. Hypoxia induced up-regulation of angiopoietin-2 in colorectal cancer. Oncol. Rep, 15, 779-783 (2006).

18. Goede, V. et al. Identification of serum angiopoietin-2 as a biomarker for clinical outcome of colorectal cancer patients treated with bevacizumab-containing therapy. Br. J. Cancer, 103, 14071414 (2010).

19. Llovet, J. M. et al. Plasma biomarkers as predictors of outcome in patients with advanced hepatocellular carcinoma. Clin. Cancer Res, 18, 2290-2300 (2012).

20. Fagin, J. A. \& Wells, S. A. Biologic and clinical perspectives on thyroid cancer. N. Engl. J. Med, 375, 105410-105467 (2016).

21. LENVIMA (Lenvatinib). Drug Interview Form (Package insert) (Eisai Co., Ltd., Tokyo, Japan, 2015).

22. Ozeki, T. et al. Influence of CYP3A4/5 and ABC transporter polymorphisms on lenvatinib plasma trough concentrations in Japanese patients with thyroid cancer. Sci. Rep, 9, 5404 (2019).

\section{Tables}

Due to technical limitations, Tables 1 and 2 are only available as a download in the Supplemental Files section.

\section{Figures}




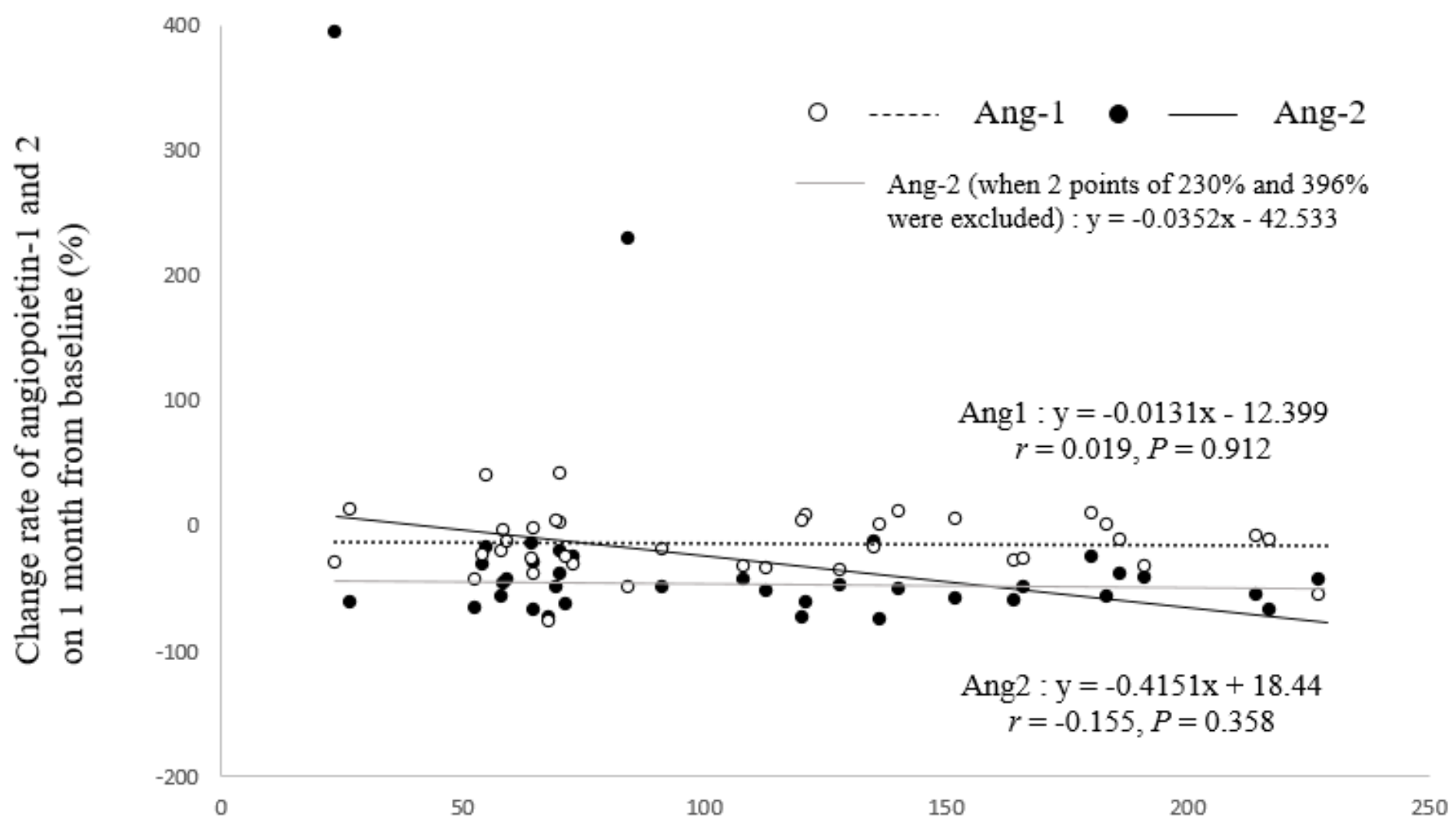

Lenvatinib plasma trough concentration $(\mathrm{ng} / \mathrm{mL})$ on day 29

\section{Figure 1}

Relationship between rate of change of angiopoietin-1 (open circles) and angiopoietin-2 (closed circles) at 1 month after initiation of lenvatinib treatment from baseline with trough plasma concentrations of lenvatinib at 1 month. 


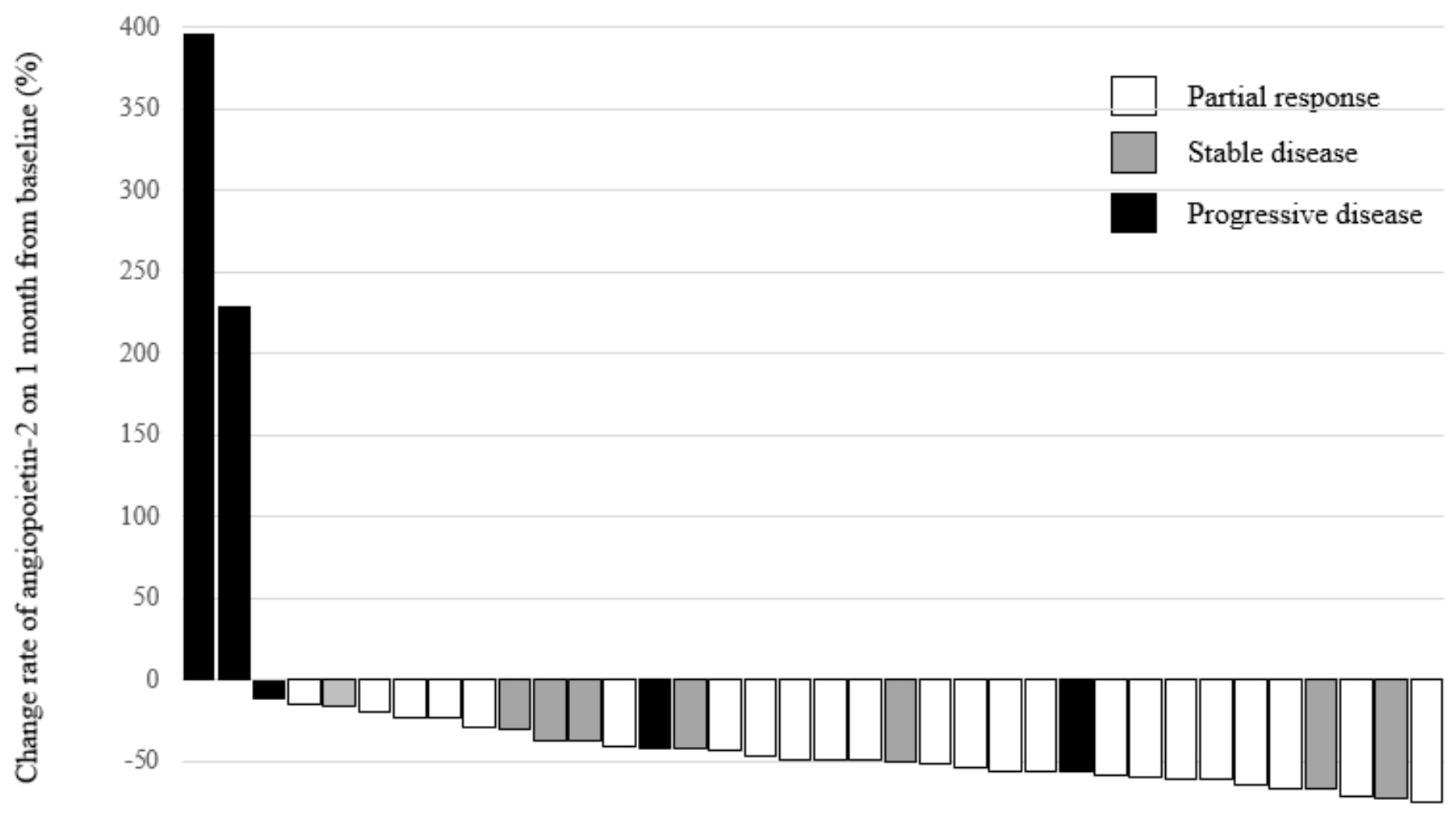

$-100$

Figure 2

Waterfall plot of the rate of change of angiopoietin-2 1 month after initiation of lenvatinib treatment from baseline in 36 patients. 


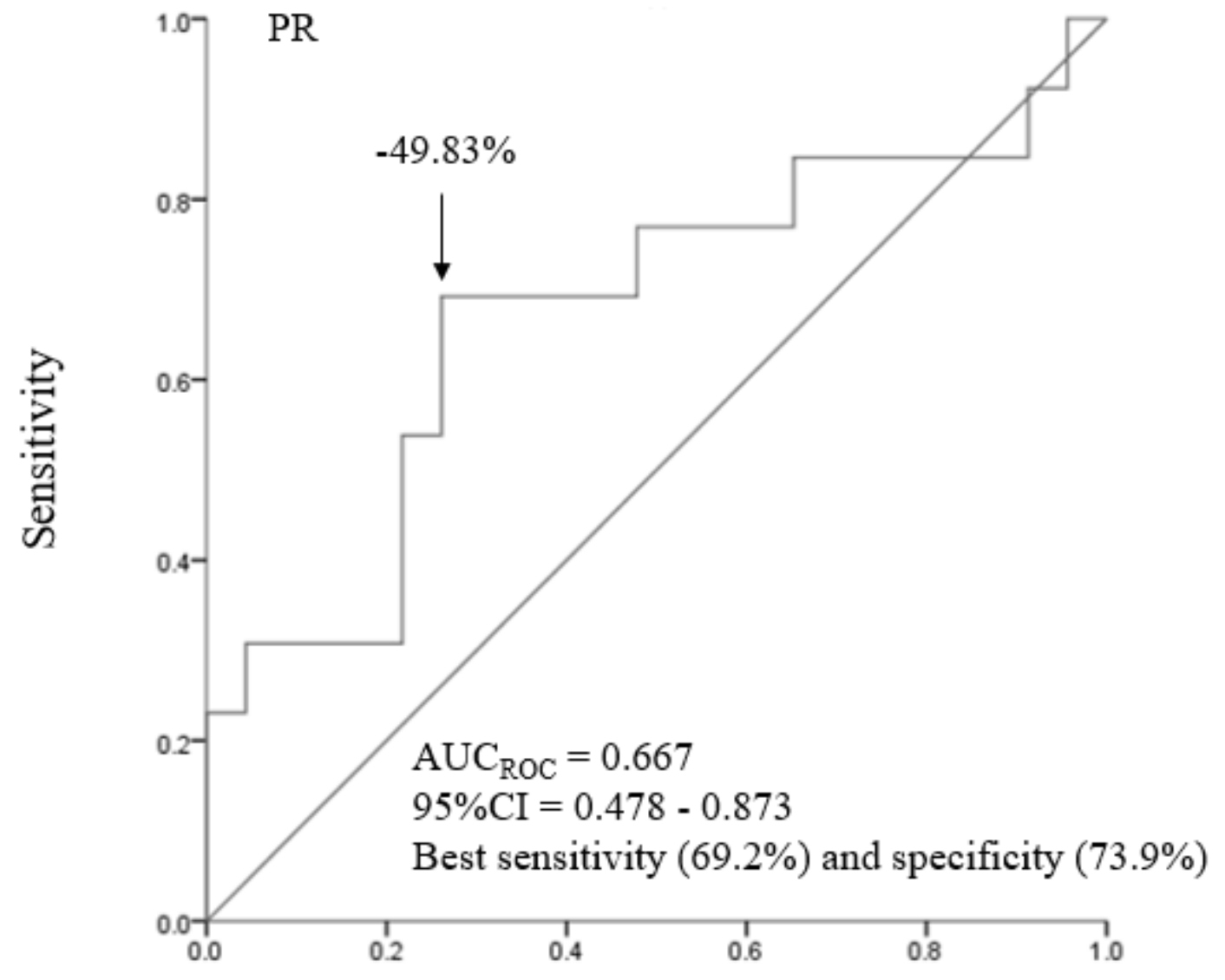

\section{1-specificity}

Figure 3

Receiver operating characteristic (ROC) analyses of the performance of the threshold of the rate of change of angiopoietin-2 in the prediction of partial response. 


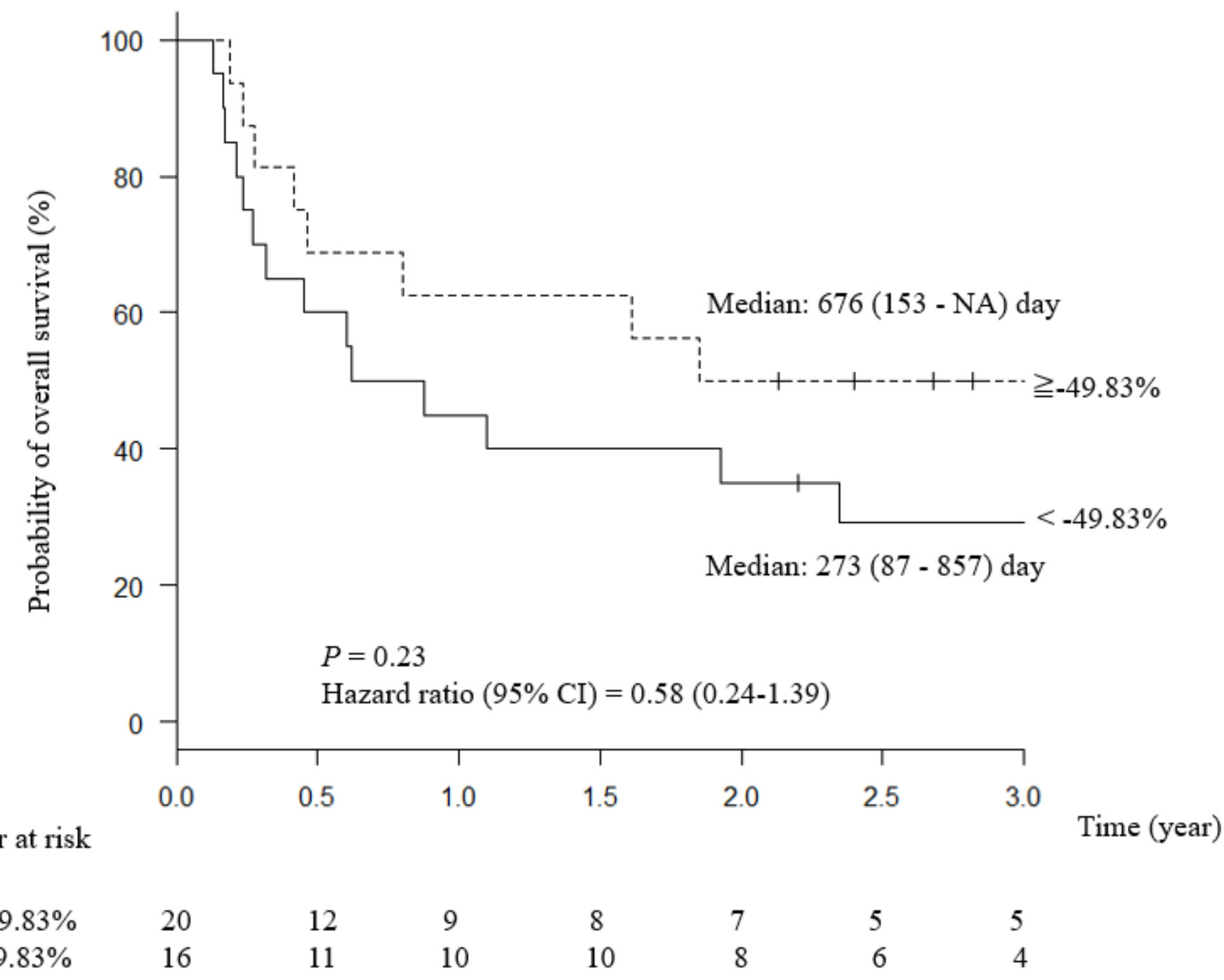

Figure 4

Kaplan-Meier curve of overall survival (OS) according to thresholds of the rate of change of angiopoietin2 of at least $-49.83 \%$ (dotted line) and less than $-49.83 \%$ (black line).

\section{Supplementary Files}

This is a list of supplementary files associated with this preprint. Click to download.

- Table1.xlsx

- Table2.xlsx 